\title{
Tendências temporais e fatores associados ao diagnóstico em estágio avançado de câncer do colo uterino: análise dos dados dos registros hospitalares de câncer no Brasil, 2000-2012* \\ doi: $10.5123 /$ S1679-49742018000200003
}

\author{
Temporal trend and associated factors to advanced stage at diagnosis of cervical cancer: \\ analysis of data from hospital based cancer registries in Brazil, 2000-2012
}

Tendencia temporal y factores asociados al diagnóstico de cáncer de cuello uterino en estado avanzado: análisis de los datos de registros hospitalarios de cáncer en Brasil, 2000-2012

\author{
Nelson Luiz Renna Junior ${ }^{1}$ - (D) orcid.org/0000-0003-1811-9504 \\ Gulnar Azevedo e Silva' \\ 'Universidade do Estado do Rio de Janeiro, Instituto de Medicina Social, Rio de Janeiro, RJ, Brasil
}

\section{Resumo}

Objetivo: analisar a tendência temporal e os fatores associados ao diagnóstico em estágio avançado de câncer do colo uterino no Brasil. Métodos: análise de séries temporais e estudo transversal com dados dos registros hospitalares de câncer (2000-2012); utilizaram-se modelos joinpoint e regressão multinomial. Resultados: analisaram-se 65.843 casos; a mediana do intervalo entre diagnóstico e tratamento foi de 59 dias; o percentual de diagnósticos em estágio avançado aumentou no período - variação anual de $1,10 \%\left(\mathrm{IC}_{95 \%} 0,80 ; 1,50\right)$; mulheres com estudo superior tiveram menores chances de estadiamento avançado, comparadas a analfabetas $\left(\mathrm{OR}=0,38 ; \mathrm{IC}_{95 \%} 0,31 ; 0,47\right)$; indígenas $\left(\mathrm{OR}=2,38 ; \mathrm{IC}_{95 \%} 1,06 ; 5,33\right)$ e negras $(\mathrm{OR}=1,16$; $\left.\mathrm{IC}_{95 \%} 1,02 ; 1,31\right)$ frente às brancas, e mulheres tratadas na região Norte $\left(\mathrm{OR}=2,55 ; \mathrm{IC}_{95 \%} 2,26 ; 2,89\right)$ frente às do Sudeste, apresentaram maiores chances; outros fatores associados positivamente ao desfecho foram 'idade', 'tipo histológico' e 'estado conjugal'. Conclusão: houve tendência crescente de diagnósticos em estágio avançado; desigualdades afetam as chances de estadiamento avançado de câncer cervical.

Palavras-chave: Neoplasias do Colo do Útero; Saúde da Mulher; Registros Hospitalares; Acesso aos Serviços de Saúde; Oncologia.

\footnotetext{
*Artigo derivado da dissertação de mestrado intitulada 'Acesso ao diagnóstico e tratamento de pacientes com câncer de mama e do colo uterino no Brasil: uma análise dos dados dos Registros Hospitalares de Câncer', defendida por Nelson Luiz Renna Junior junto ao Programa de Pós-Graduação em Saúde Coletiva do Instituto de Medicina Social da Universidade do Estado do Rio de Janeiro (UERJ) em 2016.
}

Endereço para correspondência:

Nelson Luiz Renna Junior - Rua São Francisco Xavier, n 524, Bloco E, $7 \circ$ andar, Maracanã, Rio de Janeiro, RJ, Brasil

E-mail:nelsonrenna@gmail.com 


\section{Introdução}

0 câncer do colo uterino ocupa lugar de destaque entre os tumores que afetam mulheres em todo 0 mundo, apresentando ocorrência frequente, principalmente em regiões menos desenvolvidas. ${ }^{1}$ Em 2012, a mortalidade por esse tipo de câncer chegava a ser 18 vezes maior em áreas dotadas de menos recursos, como a África Central, quando comparadas a países de alta renda, como os da Europa Ocidental. No mesmo ano, ele era o quarto tipo de câncer mais comum em mulheres no mundo, com $85 \%$ dos casos em países de média e baixa renda. ${ }^{1}$

No Brasil, foram estimados 16.340 casos novos em 2016, com incidência de 15,85 casos para cada 100 mil mulheres. ${ }^{2}$ Era 0 câncer mais comum entre mulheres na região Norte do país e o segundo em incidência nas regiões Centro-Oeste e Nordeste. ${ }^{2} \mathrm{~A}$ taxa de mortalidade nacional padronizada por idade, estimada para 2012, foi de 7,3 por 100 mil mulheres. ${ }^{1}$

A aplicação de rastreamento organizado pode levar a uma redução da ordem de $80 \%$ na mortalidade pela doença, como observado em países de alta renda desde 0 início do século XX. ${ }^{3}$ Nos países de média e baixa renda, os resultados são menos expressivos devido a limitações de acesso à saúde, como baixa cobertura de programas de rastreamento e atrasos na realização de procedimentos diagnósticos e terapêuticos. ${ }^{4,5}$

\section{A aplicação de rastreamento organizado pode levar a uma redução da ordem de $80 \%$ na mortalidade por câncer no colo uterino.}

Com o objetivo de garantir atendimento integral a indivíduos com diagnóstico de câncer, o Ministério da Saúde do Brasil, por meio da Portaria GM/MS $\mathrm{n}^{0} 2.439$, de 8 de dezembro de 2005 , estabeleceu a Política Nacional de Atenção Oncológica, enfatizando a importância da prevenção, diagnóstico precoce e tratamento oportuno como medidas para reduzir a incidência e a mortalidade pela doença. ${ }^{6}$

Estudos recentes abordaram o risco de diagnóstico em estágio avançado e o tempo para início do tratamento no Brasil $, 7,8$ trazendo à tona o debate sobre tendência de elevação do percentual de diagnósticos em estágio avançado mesmo em um contexto de aumento da disponibilidade do rastreamento no país, especialmente a partir da década de $1990 .^{5}$ Assim, torna-se cada vez mais pertinente o estudo dos determinantes do estadiamento da doença no momento do diagnóstico.

0 presente estudo teve como objetivo analisar a tendência temporal e os fatores associados ao diagnóstico em estágio avançado de câncer do colo uterino no Brasil.

\section{Métodos}

Foi realizada análise de série temporal baseada em estudo da tendência de evolução dos percentuais de mulheres com câncer de colo uterino em estágios III e IV no momento do diagnóstico, e estudo transversal para análise das características epidemiológicas e clínicas associadas ao estadiamento avançado.

0 conjunto de dados relativo às unidades de saúde (clínicas e hospitais que realizam atendimento oncológico) de todo o país, exceto as situadas no estado de São Paulo, foi obtido por meio do integrador do Registro Hospitalar de Câncer (RHC), disponível no sítio do Instituto Nacional de Câncer José Alencar Gomes da Silva (INCA) na internet (https://irhc.inca.gov.br/RHCNet). Os dados das unidades paulistas foram obtidos em consulta ao endereço da Fundação Oncocentro de São Paulo (FOSP) na internet (http://www.fosp.saude. sp.gov.br/publicacoes/acessobancodados). 0 acesso aos sítios eletrônicos para obtenção dos dados deu-se em 21 de janeiro de 2016.

A população de estudo foi composta por casos de câncer de colo do útero (código C53 da 10ª Revisão da Classificação Estatística Internacional de Doenças e Problemas Relacionados à Saúde - CID-10) registrados pelos RHC, com diagnóstico entre os anos de 2000 e 2012, com confirmação histopatológica, 18 anos ou mais de idade na data do diagnóstico, e com informações sobre estadiamento disponíveis.

Foram elegíveis os casos referentes a tumores invasivos com confirmação histopatológica. Foram excluídos todos os casos de neoplasias in situ ou intraepiteliais, assim como tumores benignos ou não invasivos. Também foram excluídos do estudo sarcomas, linfomas e tumores neuroendócrinos, por se tratarem de entidades cuja ocorrência, diagnóstico, fatores de risco e tratamento são distintos daqueles referentes à patologia objeto deste estudo. 
Foram incluídos apenas casos classificados pelo RHC como analíticos (isto é, casos para os quais o tratamento principal foi realizado na instituição responsável pelo registro), para minimizar a ocorrência de registros em duplicidade, uma vez que um determinado caso poderia ser inserido nas planilhas por instituições diferentes (ora como analítico, ora como não analítico) sendo, portanto, registrado mais de uma vez.

Foram calculadas medidas de tendência central $\mathrm{e}$ dispersão para os intervalos - em dias - entre a data do diagnóstico (data do laudo histopatológico) e a data do início do primeiro tratamento específico para a doença, como também entre a data da primeira consulta na instituição e 0 início do tratamento. Outrossim, foi registrado o percentual de casos cujo tempo até a instituição do tratamento específico superou 60 dias.

Os casos nos quais a Unidade da Federação (UF) de nascimento da paciente era diferente daquela da unidade hospitalar que efetuou o registro foram considerados nas análises com o objetivo de alcançar aproximação de uma situação hipotética de barreira ao acesso - situação em que a usuária precisaria se dirigir a uma localidade diferente daquela de sua residência, na busca por atendimento.

As variáveis de estudo incluíram:

- escolaridade (nenhuma; ensino fundamental incompleto; ensino fundamental completo; ensino médio; ensino superior);

- região da unidade hospitalar (UF na qual está localizada a instituição de saúde que realizou o registro); - região de nascimento (UF de nascimento da pessoa); - raça/cor da pele (branca; parda; indígena; amarela; preta);

- estado conjugal (casada/união estável; solteira; separada; viúva);

- idade (em faixas etárias, sendo a primeira composta por mulheres com menos de 30 anos; as demais categorias foram definidas por intervalos de $5 \mathrm{em} 5$ anos, até 70 ou mais anos);

- diagnóstico e tratamento prévios (com diagnóstico, com tratamento; com diagnóstico, sem tratamento; sem diagnóstico, sem tratamento);

- tipo histológico (diferenciação ductal, lobular e outros, incluindo tumores que não se enquadraram nas categorias anteriores);

- completude do tratamento (sim; não); - história familiar de câncer (presença; ausência);
- tabagismo (nunca fumou; ex-tabagista; tabagista atual); - etilismo (nuncafezuso de álcool; ex-etilista; etilista atual). ${ }^{?}$

Conforme a classificação da Federação Internacional de Ginecologia e Obstetrícia (FIGO) para estadiamento,${ }^{10} 0$ câncer de colo uterino estágio I é aquele que se encontra restrito ao útero. Os tumores no estágio II são os que se disseminaram para além do útero sem, contudo, atingir os ossos da pelve ou 0 terço inferior da vagina. Os tumores em estágio III são os que acometem a parede pélvica, o terço inferior da vagina, ou que causam obstrução utereral. 0 estágio IV inclui os tumores que invadem a mucosa do reto ou da bexiga, ou que se disseminam a distância. Os casos foram classificados de acordo com o estágio no momento do diagnóstico. No grupo 1 (doença inicial), foram incluídos aqueles com estágio I; no grupo 2 (doença localizada), aqueles com estágio II; e no grupo 3 (doença avançada), aqueles com estágios III e IV.

Para estudo da tendência temporal do estadiamento ao longo do período de observação, utilizou-se modelo de regressão joinpoint, tendo como desfecho o estadiamento no diagnóstico (expresso como percentual de estágios III e IV), e como variável independente 0 ano do diagnóstico. Para o ajuste do modelo, foram admitidos de 0 a 3 joinpoints (tendências apresentadas pelos segmento de reta) e estimadas as variações percentuais anuais com intervalos de confiança de $95 \%$ $\left(\mathrm{IC}_{95 \%}\right) .{ }^{11} \mathrm{O}$ aplicativo Joinpoint versão 4.2.02 (2015) foi empregado nas análises de tendência temporal.

Para o estudo transversal, utilizou-se modelo de regressão logística multinomial; os casos classificados como doença localizada e doença avançada foram comparados aos classificados como doença inicial (referência). Inicialmente, foi realizada análise bruta por meio de modelo de regressão logística multinomial e teste de Wald. Foram selecionadas para o modelo múltiplo as variáveis que apresentaram associação com o desfecho de valor $\mathrm{p} \leq 0,20^{12}$ e que detinham no máximo $35 \%$ de registros sem informação. Foram calculadas as razões de chances (ou odds ratios - $\mathrm{OR}$ ) brutas e ajustadas, e seus respectivos $\mathrm{IC}_{95 \%}$.

Com o intuito de analisar a relação entre estágio no diagnóstico e histórico de tabagismo, etilismo, estado conjugal, raça/cor da pele e história familiar de câncer, realizou-se uma segunda análise com a mesma metodologia, porém excluindo-se os casos oriundos das unidades localizadas no estado de São Paulo, uma vez que as informações relativas a essas 
variáveis não estavam disponíveis no conjunto de dados obtidos da FOSP.

As análises foram realizadas utilizando-se 0 aplicativo R versão 3.0.3 de 2014; os coeficientes dos modelos de regressão foram extraídos com auxílio do pacote Texreg. ${ }^{13}$ Todas as planilhas foram editadas utilizando-se o aplicativo Apache OpenOffice 4.1.1 de 2014.

0 estudo foi aprovado pelo Comitê de Ética em Pesquisa do Instituto de Medicina Social da Universidade do Estado do Rio de Janeiro, conforme a Resolução do Conselho Nacional de Saúde no 196, de 10 de outubro de 1996, mediante Certificado de Apresentação para Apreciação Ética n ${ }^{0} 50685115.9 .0000 .5260$, de 28 de outubro de 2015.

\section{Resultados}

No total, 65.843 mulheres atenderam aos critérios de inclusão no estudo. A idade mediana no diagnóstico foi de 52 anos (18 a 111 anos), 17\% eram analfabetas e $36 \%$ tinham ensino fundamental incompleto. Não havia informação sobre escolaridade para $26 \%$ dos casos (Tabela 1).

0 tempo entre a data do diagnóstico e a data do início do tratamento variou de 0 a 180 dias (mediana de 59 dias). 0 intervalo entre a primeira consulta e 0 início do tratamento variou de 0 a 182 dias (mediana de 36 dias). Em 49,20\% dos casos, o tempo entre diagnóstico e início do tratamento superou 60 dias.

As mulheres de 45 a 49 anos representaram $13,05 \%$ dos casos. A região Sudeste concentrou 45,90\%, seguida pelas regiões Nordeste $(31,20 \%)$, Sul $(11,34 \%)$, Norte $(9,70 \%)$ e Centro-Oeste (1,89\%). Para $25 \%$ das mulheres, o tratamento foi realizado em UF diferente daquela do nascimento.

Completaram o tratamento inicial proposto $83,77 \%$ das mulheres, das quais $17,30 \%$ se encontravam sem evidência da doença ao fim dele. A variável 'estado da doença ao final do tratamento' continha $42,30 \%$ das observações sem informação. A maioria das mulheres $(69,19 \%)$ chegou à instituição de referência com diagnóstico firmado e apenas 9,86\% já tinham recebido algum tipo de tratamento. As características das mulheres estudadas são mostradas nas Tabelas 1 e 2.

0 percentual de diagnósticos em estágios III e IV aumentou significativamente, de 2000 (46,80\%) a $2012(53,53 \%)$ : variação percentual anual de 1,10 ; $\mathrm{IC}_{95 \%} 0,80 ; 1,50$ (Figura 1).
As chances de ter diagnóstico da doença nas categorias localizada e avançada aumentaram com a idade, sendo que a diferença se tornou estatisticamente significativa a partir da faixa de 40 a 44 anos, tanto para doença localizada $\left(\mathrm{OR}=1,27 ; \mathrm{IC}_{95 \%} 1,09 ; 1,47\right)$ como para doença avançada $\left(\mathrm{OR}=1,15 ; \mathrm{IC}_{95 \%}, 1,00 ; 1,32\right)(\mathrm{Ta}-$ bela 3). Quando comparadas às mulheres com até 30 anos, mulheres de 65 a 69 anos tiveram maiores chances de apresentar doença localizada $\left(\mathrm{OR}=2,40 ; \mathrm{IC}_{95 \%}\right.$ $2,04 ; 2,82)$ e avançada $\left(\mathrm{OR}=2,09 ; \mathrm{IC}_{95 \%} 1,79 ; 2,44\right)$. Mulheres com ensino superior apresentaram menores chances de desenvolver doença localizada $(0 \mathrm{R}=0,65$; $\left.\mathrm{IC}_{95 \%} 0,54 ; 0,77\right)$, assim como de desenvolver doença avançada $\left(\mathrm{OR}=0,45 ; \mathrm{IC}_{95 \%} 0,39 ; 0,53\right)$.

0 modelo ajustado foi composto por todas as variáveis incluídas na análise bruta. Comparadas às mulheres da região Sudeste, mulheres das demais regiões apresentaram chances maiores de desenvolver doença localizada e doença avançada, no diagnóstico (Tabela 4).

As mulheres que nasceram em UF diferente daquela na qual foi realizado o tratamento apresentaram menores chances de desenvolver doença avançada $(\mathrm{OR}=0,91$; $\left.\mathrm{IC}_{95 \%} 0,86 ; 0,96\right)$, sem diferença significativa para a categoria localizada. As mulheres portadoras de adenocarcinomas tiveram chances menores de apresentar doença localizada $\left(\mathrm{OR}=0,63 ; \mathrm{IC}_{95 \%} 0,59 ; 0,68\right)$ e avança$\mathrm{da}\left(\mathrm{OR}=0,48 ; \mathrm{IC}_{95 \%} 0,44 ; 0,51\right)$ quando comparadas às portadoras de tumores de origem escamosa (Tabela 3).

$\mathrm{Na}$ segunda análise, excluindo-se os 8.968 casos oriundos do estado de São Paulo, foram acrescentadas apenas as variáveis 'raça/cor da pele' e 'estado civil', porque atenderam aos critérios estabelecidos para inclusão na análise múltipla. As variáveis 'tabagismo', 'etilismo' e 'história familiar de câncer' não foram incluídas por apresentarem mais de 35\% de dados faltantes. Mulheres pardas tiveram menores chances de apresentar doença localizada $\left(\mathrm{OR}=0,91 ; \mathrm{IC}_{95 \%}\right.$ $0,84 ; 0,98)$ quando comparadas às brancas. As indígenas apresentaram as maiores chances de diagnóstico na categoria avançada $\left(\mathrm{OR}=2,38 ; \mathrm{IC}_{95 \%}, 1,06 ; 5,33\right)$, como também maiores chances apresentaram as negras $\left(\mathrm{OR}=1,16 ; \mathrm{IC}_{95 \%} 1,02 ; 1,31 ;\right)$, em comparação às brancas. Na Tabela 4 estão apresentados os resultados da segunda análise, que acrescentou às variáveis de estudo a 'raça/cor da pele' e o 'estado conjugal'.

Mulheres viúvas apresentaram chances maiores de desenvolver doença localizada $\left(\mathrm{OR}=1,11 ; \mathrm{IC}_{95 \%}\right.$ 
Tabela 1 - Características sociodemográficas das mulheres com diagnóstico de câncer de colo uterino que atenderam aos critérios de inclusão no estudo, segundo os registros hospitalares de câncer, Brasil, 2000-2012

\begin{tabular}{|c|c|c|}
\hline Características sociodemográficas & N & $\%$ \\
\hline \multicolumn{3}{|l|}{ Escolaridade } \\
\hline Nenhuma & 11.156 & 16,94 \\
\hline Ensino fundamental incompleto & 23.728 & 36,04 \\
\hline Ensino fundamental completo & 8.075 & 12,26 \\
\hline Ensino médio & 4.706 & 7,15 \\
\hline Ensino superior & 1.053 & 1,60 \\
\hline Sem informação & 17.125 & 26,01 \\
\hline \multicolumn{3}{|l|}{ Região da unidade hospitalar } \\
\hline Centro-0este & 1.243 & 1,89 \\
\hline Nordeste & 20.547 & 31,21 \\
\hline Norte & 6.371 & 9,68 \\
\hline Sudeste & 30.215 & 45,89 \\
\hline Sul & 7.467 & 11,34 \\
\hline \multicolumn{3}{|l|}{ Região de nascimento } \\
\hline Centro-0este & 1.265 & 2,01 \\
\hline Nordeste & 26.335 & 41,86 \\
\hline Norte & 5.617 & 8,93 \\
\hline Sudeste & 22.579 & 35,89 \\
\hline Sul & 6.952 & 11,05 \\
\hline Exterior & 160 & 0,25 \\
\hline \multicolumn{3}{|c|}{ Região de nascimento difere da Unidade da Federação onde se encontra a unidade hospitalar } \\
\hline Não & 46.058 & 69,95 \\
\hline Sim & 16.690 & 25,35 \\
\hline Sem informação & 3.095 & 4,70 \\
\hline \multicolumn{3}{|l|}{ Tabagismo } \\
\hline Ex-tabagista & 1.401 & 2,86 \\
\hline Nunca & 18.815 & 38,35 \\
\hline Sim & 11.261 & 22,95 \\
\hline Sem informação & 17.583 & 35,84 \\
\hline \multicolumn{3}{|l|}{ Etilismo } \\
\hline Ex-etilista & 537 & 1,09 \\
\hline Nunca & 23.256 & 47,40 \\
\hline Sim & 3.792 & 7,73 \\
\hline Sem informação & 21.475 & 43,77 \\
\hline \multicolumn{3}{|l|}{ Estado conjugal } \\
\hline Solteira & 12.875 & 26,24 \\
\hline Casada & 22.155 & 45,16 \\
\hline União consensual & 359 & 0,73 \\
\hline Separada judicialmente & 2.762 & 6,04 \\
\hline Viúva & 2.762 & 5,63 \\
\hline Sem informação & 3.357 & 6,84 \\
\hline \multicolumn{3}{|l|}{ Raça/cor da pele } \\
\hline Amarela & 460 & 0,94 \\
\hline Branca & 15.972 & 32,56 \\
\hline Indígena & 143 & 0,29 \\
\hline Parda & 24.947 & 50,85 \\
\hline Preta & 2.947 & 6,01 \\
\hline Sem informação & 4.591 & 9,36 \\
\hline
\end{tabular}


Tabela 2 - Características clínicas e relacionadas a diagnóstico e tratamento das mulheres com diagnóstico de câncer de colo uterino que atenderam aos critérios de inclusão no estudo, segundo os registros hospitalares de câncer, Brasil, 2000-2012

\begin{tabular}{|c|c|c|}
\hline Características clínicas e relacionadas a diagnóstico e tratamento & $\mathbf{N}$ & $\%$ \\
\hline \multicolumn{3}{|l|}{ Tratamento na instituição } \\
\hline Completou tratamento & 55.155 & 83,77 \\
\hline Não completou tratamento & 3.800 & 5,77 \\
\hline Sem informação & 6.888 & 10,46 \\
\hline \multicolumn{3}{|l|}{ Estado da doença ao final do tratamento } \\
\hline Sem evidência da doença & 11.390 & 17,30 \\
\hline Remissão parcial & 1.673 & 2,54 \\
\hline Doença estável & 12.156 & 18,46 \\
\hline Doença em progressão & 3.193 & 4,85 \\
\hline Fora de possibilidade terapêutica & 618 & 0,94 \\
\hline Óbito & 6.183 & 9,39 \\
\hline Não se aplica & 2.778 & 4,22 \\
\hline Sem informação & 27.852 & 42,30 \\
\hline \multicolumn{3}{|l|}{ História familiar de câncer } \\
\hline Não & 17.497 & 35,66 \\
\hline Sim & 9.846 & 20,07 \\
\hline Sem informação & 21.717 & 44,27 \\
\hline \multicolumn{3}{|l|}{ Diagnóstico e tratamento prévios } \\
\hline Com diagnóstico, com tratamento & 6.492 & 9,86 \\
\hline Com diagnóstico, sem tratamento & 39.064 & 59,33 \\
\hline Sem diagnóstico, sem tratamento & 18.939 & 28,76 \\
\hline Sem informação & 25.431 & 38,62 \\
\hline \multicolumn{3}{|l|}{ Tipo histológico } \\
\hline Adenocarcinoma & 7.651 & 11,62 \\
\hline Escamoso & 54.351 & 82,55 \\
\hline Adenoescamoso & 803 & 1,22 \\
\hline Neoplasia invasiva, sem especificação & 3.038 & 4,61 \\
\hline \multicolumn{3}{|l|}{ Estágio no diagnóstico } \\
\hline 1 & 14.024 & 21,30 \\
\hline II & 21.293 & 32,34 \\
\hline III & 25.782 & 39,16 \\
\hline IV & 4.744 & 7,21 \\
\hline
\end{tabular}

$1,01 ; 1,23)$ quando comparadas às casadas. As separadas apresentaram maiores chances de doença avançada $\left(\mathrm{OR}=1,29 ; \mathrm{IC}_{95 \%} 1,13 ; 1,46\right)$, assim como as solteiras $\left(\mathrm{OR}=1,46 ; \mathrm{IC}_{95 \%} 1,36 ; 1,57\right)$ e as viúvas $\left(\mathrm{OR}=1,34 ; \mathrm{IC}_{95 \%}\right.$ $1,22 ; 1,48)$, comparativamente às casadas.

\section{Discussão}

O diagnóstico do câncer de colo uterino no Brasil ocorreu tardiamente em mais da metade dos casos e mulheres com menor nível socioeconômico apresen- taram maiores chances de serem diagnosticadas em estágios avançados. Indígenas e negras apresentaram associação significativa com estadiamento avançado no diagnóstico, na comparação com mulheres brancas. Mulheres tratadas na região Sudeste apresentaram chances significativamente menores de terem diagnóstico tardio, na comparação com as demais macrorregiões do país. Após o diagnóstico, o tempo para início do tratamento foi superior a 60 dias para mais da metade das mulheres.

0 presente estudo apresenta limitações a destacar. Os dados foram obtidos de base secundária, restringindo-se 


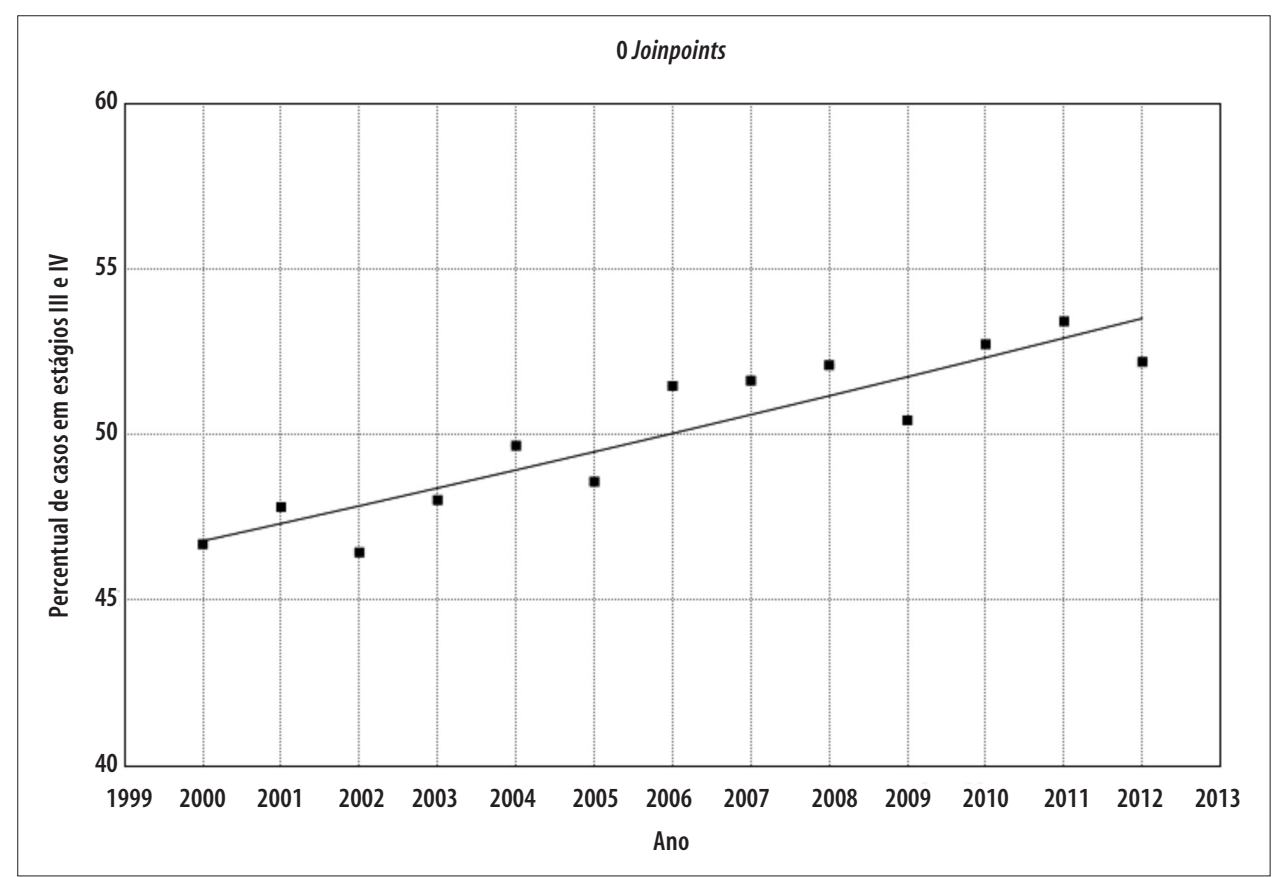

Figura 1 - Evolução temporal do percentual de casos de mulheres com diagnóstico de câncer de colo uterino em estágios III e IV, segundo os registros hospitalares de câncer, Brasil, 2000-2012

as análises às informações disponibilizadas pelo RHC. Assim, aspectos importantes relacionados à história natural da doença não puderam ser analisados de forma direta. A baixa completude encontrada em algumas variáveis impossibilitou sua inclusão na análise. 0 RHC reúne informações de casos atendidos na grande maioria das unidades de saúde do país, estando presente em $91 \%$ das unidades credenciadas pelo Sistema Único de Saúde (SUS), com cobertura crescente nos últimos anos. ${ }^{9,14}$

Considerando-se o fato de as informações do RHC estarem sujeitas a atualização constante e dada a possibilidade de sub-registro em unidades de saúde situadas em regiões dotadas de menos recursos, cumpre ressaltar que parte do efeito encontrado nas tendências temporais pode-se dever ao registro incompleto por parte das instituições de saúde. É possível sustentar, entretanto, que o sub-registro, majoritariamente oriundo de regiões mais carentes de recursos, afetaria principalmente os casos mais avançados. Com isso, o aumento percentual de diagnósticos em estágios III e IV poderia ser ainda maior do que o reportado no presente trabalho se os casos não notificados incluíssem, em sua maioria, mulheres com diagnóstico em fase avançada.
0 número total de casos registrados foi pequeno quando comparado à estimativa de incidência anual da doença, mesmo levando-se em consideração 0 aumento da cobertura nos últimos anos. Isso sugere que o sistema passa por constante atualização, com aumento progressivo de sua cobertura. Ademais, o RHC cobre principalmente usuários do SUS, deixando de fora a quase totalidade das pessoas cobertas pelo sistema suplementar. Este fato poderia se traduzir em viés de seleção caso a distribuição do desfecho entre as usuárias do sistema suplementar fosse substancialmente diferente da observada na amostra estudada.

No ano de 2012, a Lei Federal $n^{0} 12.732^{15}$ estabeleceu como prazo máximo para início do tratamento de câncer o limite de 60 dias após o diagnóstico. Embora a amostra do presente estudo tenha revelado os tempos medianos entre diagnóstico e início do tratamento abaixo do tempo recomendado por lei, em quase metade dos casos o limite foi excedido. Isto pode explicar, em conjunção com outros fatores - como barreiras de acesso ao tratamento e características biológicas da doença -, o elevado percentual de mulheres com doença persistente ao fim do tratamento. Fica clara a necessidade da adoção de medidas capazes de reduzir barreiras e garantir pronto tratamento dos casos 
diagnosticados, contribuindo para que os benefícios relacionados aos programas de rastreamento estejam disponíveis a um número cada vez maior de mulheres.

Observou-se aumento do percentual de diagnósticos em estágios III e IV, ao longo do período compreendido entre os anos 2000 e 2012. Este achado é importante por sugerir que as políticas de atenção oncológica não têm sido capazes de propiciar diagnóstico precoce, capaz de garantir não apenas melhor prognóstico como também redução da incidência da doença mediante tratamento de lesões préinvasivas. ${ }^{16}$ Mulheres sintomáticas não captadas pelo rastreamento, possivelmente, perdem oportunidades de diagnóstico durante passagens pouco resolutivas pelos serviços da atenção primária e secundária, resultando na chegada à atenção oncológica já em

\section{Tabela 3 - Associação entre características sociodemográficas e clínicas de mulheres com câncer de colo uterino e 0 estadiamento avançado da doença, Brasil, 2000-2012}

\begin{tabular}{|c|c|c|c|c|}
\hline \multirow[b]{2}{*}{ Características demográficas e clínicas } & \multicolumn{2}{|c|}{ Doença localizada } & \multicolumn{2}{|c|}{ Doença avançada } \\
\hline & $\begin{array}{c}\text { Análise bruta } \\
\text { OR }^{\mathrm{a}}\left(\mathrm{IC}_{95 \%}{ }^{\mathrm{b}}\right)\end{array}$ & 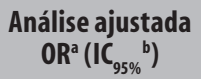 & $\begin{array}{c}\text { Análise bruta } \\
\text { OR }^{\mathrm{a}}\left(\mathrm{IC}_{95 \%}{ }^{\mathrm{b}}\right)\end{array}$ & $\begin{array}{c}\text { Análise ajustada } \\
\operatorname{OR}^{\mathrm{a}}\left(\mathrm{IC}_{95 \%}{ }^{b}\right)\end{array}$ \\
\hline \multicolumn{5}{|l|}{ Idade (em anos) } \\
\hline$<30$ & 1,00 & 1,00 & 1,00 & 1,00 \\
\hline $30-34$ & $1,06(0,93 ; 1,22)$ & $1,01(0,86 ; 1,18)$ & $0,94(0,83 ; 1,06)$ & $0,90(0,78 ; 1,05)$ \\
\hline $35-39$ & $1,15(1,01 ; 1,31)$ & $1,11(0,96 ; 1,30)$ & $1,01(0,89 ; 1,13)$ & $0,98(0,85 ; 1,13)$ \\
\hline $40-44$ & $1,33(1,18 ; 1,51)$ & $1,27(1,09 ; 1,47)$ & $1,21(1,08 ; 1,36)$ & $1,15(1,00 ; 1,32)$ \\
\hline $45-49$ & $1,44(1,27 ; 1,63)$ & $1,46(1,26 ; 1,69)$ & $1,51(1,35 ; 2,70)$ & $1,51(1,32 ; 1,73)$ \\
\hline $50-54$ & $1,81(1,59 ; 2,05)$ & $1,80(1,55 ; 2,09)$ & $1,96(1,74 ; 2,20)$ & $1,84(1,60 ; 2,12)$ \\
\hline $55-59$ & $2,00(1,76 ; 2,27)$ & $1,94(1,66 ; 2,25)$ & $2,05(1,83 ; 2,31)$ & $1,89(1,64 ; 2,18)$ \\
\hline $60-64$ & $2,11(1,85 ; 2,40)$ & $1,96(1,68 ; 2,29)$ & $2,14(1,89 ; 2,41)$ & $1,86(1,61 ; 2,15)$ \\
\hline $65-69$ & $2,35(2,05 ; 2,69)$ & $2,40(2,04 ; 2,82)$ & $2,23(1,57 ; 2,53)$ & $2,09(1,79 ; 2,44)$ \\
\hline$\geq 70$ & $2,46(2,17 ; 2,79)$ & $2,38(2,05 ; 2,78)$ & $2,72(2,42 ; 3,06)$ & $2,35(2,04 ; 2,71)$ \\
\hline \multicolumn{5}{|l|}{ Escolaridade } \\
\hline Nenhuma & 1,00 & 1,00 & 1,00 & 1,00 \\
\hline Ensino fundamental incompleto & $0,66(0,62 ; 0,71)$ & $0,86(0,80 ; 0,93)$ & $0,60(0,56 ; 0,64)$ & $0,73(0,68 ; 0,78)$ \\
\hline Ensino fundamental completo & $0,60(0,56 ; 0,66)$ & $0,88(0,80 ; 0,96)$ & $0,53(0.49 ; 0,58)$ & $0,69(0,63 ; 0,75)$ \\
\hline Ensino médio & $0,46(0,34 ; 0,40)$ & $0,76(0,69 ; 0,84)$ & $0,37(0,34 ; 0,40)$ & $0,53(0,48 ; 0,59)$ \\
\hline Ensino superior & $0,37(0,31 ; 0,43)$ & $0,65(0,54 ; 0,77)$ & $0,31(0,26 ; 0,36)$ & $0,45(0,39 ; 0,53)$ \\
\hline \multicolumn{5}{|l|}{ Região do hospital } \\
\hline Sudeste & 1,00 & 1,00 & 1,00 & 1,00 \\
\hline Centro-0este & $1,51(1,09 ; 1,78)$ & $1,63(1,32 ; 2,00)$ & $1,48(1,27 ; 1,73)$ & $1,29(1,06 ; 1,58)$ \\
\hline Nordeste & $2,13(2,03 ; 2,24)$ & $1,88(1,77 ; 2,00)$ & $1,67(1,59 ; 1,75)$ & $1,32(1,25 ; 1,40)$ \\
\hline Norte & $2,31(2,13 ; 2,49)$ & $2,48(2,28 ; 2,71)$ & $1,55(1,44 ; 1,62)$ & $1,63(1,50 ; 1,78)$ \\
\hline Sul & $1,46(1,35 ; 1,57)$ & $1,49(1,35 ; 1,65)$ & $1,74(1,62 ; 1,86)$ & $1,71(1,55 ; 1,87)$ \\
\hline \multicolumn{5}{|l|}{ Unidade da Federação (UF) de nascimento } \\
\hline Igual à UF do tratamento & 1,00 & 1,00 & 1,00 & 1,00 \\
\hline Difere da UF do tratamento & $0,89(0,85 ; 0,93)$ & $0,98(0,92 ; 1,03)$ & $0,86(0,82 ; 0,90)$ & $0,91(0,86 ; 0,96)$ \\
\hline \multicolumn{5}{|l|}{ Tipo histológico } \\
\hline Epidermoides & 1,00 & 1,00 & 1,00 & 1,00 \\
\hline Adenocarcinomas & $0,58(0,54 ; 0,61)$ & $0,63(0,59 ; 0,68)$ & $0,45(0,43 ; 0,48)$ & $0,48(0,44 ; 0,51)$ \\
\hline Outros & $0,79(0,72 ; 0,87)$ & $0,83(0,74 ; 0,94)$ & $1,08(0,99 ; 1,18)$ & $1,15(1,03 ; 1,29)$ \\
\hline \multicolumn{5}{|l|}{ Ano do diagnóstico } \\
\hline $2000-2005$ & 1,00 & 1,00 & 1,00 & 1,00 \\
\hline 2006-2012 & $0,97(0,93 ; 1,01)$ & $1,03(0,98 ; 1,09)$ & $1,15(1,11 ; 1,20)$ & $1,26(1,20 ; 1,33)$ \\
\hline
\end{tabular}

a) $O R$ : odds ratio: razão de chances, calculada tendo a categoria 'doença inicial' como referência.

b) $\mathcal{I C}_{95 \%}$ : intervalo de confiança de $95 \%$. 
Tabela 4 - Associação entre características sociodemográficas e clínicas de mulheres com câncer de colo uterino e 0 estadiamento avançado da doença, com a inclusão das variáveis 'raça/cor da pele' e 'estado conjugal' e exclusão dos dados das unidades de saúde do estado de São Paulo, Brasil, 2000-2012

\begin{tabular}{|c|c|c|c|c|}
\hline \multirow[b]{2}{*}{ Características demográficas e clínicas } & \multicolumn{2}{|c|}{ Doença localizada } & \multicolumn{2}{|c|}{ Doença avançada } \\
\hline & $\begin{array}{c}\text { Análise bruta } \\
\text { OR }^{\mathrm{a}}\left(\mathrm{IC}_{95 \%}^{\mathrm{b}}\right)\end{array}$ & $\begin{array}{c}\text { Análise ajustada } \\
\operatorname{OR}^{\mathrm{a}}\left(\mathrm{IC}_{95 \%}{ }^{b}\right)\end{array}$ & $\begin{array}{c}\text { Análise bruta } \\
\text { OR }^{\mathrm{a}}\left(\mathrm{IC}_{95 \%}^{\mathrm{b}}\right)\end{array}$ & $\begin{array}{c}\text { Análise ajustada } \\
\operatorname{OR}^{\mathrm{a}}\left(\mathrm{IC}_{95 \%}{ }^{\mathrm{b}}\right)\end{array}$ \\
\hline \multicolumn{5}{|l|}{ Idade (em anos) } \\
\hline$<30$ & 1,00 & 1,00 & 1,00 & 1,00 \\
\hline $30-34$ & $1,03(0,88 ; 1,20)$ & $1,04(0,86 ; 1,26)$ & $0,91(0,78 ; 1,06)$ & $0,99(0,82 ; 1,19)$ \\
\hline $35-39$ & $1,04(0,90 ; 1,21)$ & $1,06(0,89 ; 1,27)$ & $0,95(0,82 ; 1,09)$ & $1,02(0,86 ; 1,21)$ \\
\hline $40-44$ & $1,17(1,01 ; 1,35)$ & $1,18(0,99 ; 1,41)$ & $1,09(0,95 ; 1,26)$ & $1,15(0,97 ; 1,36)$ \\
\hline $45-49$ & $1,35(1,17 ; 1,57)$ & $1,47(1,24 ; 1,76)$ & $1,46(1,28 ; 1,68)$ & $1,65(1,39 ; 1,95)$ \\
\hline $50-54$ & $1,66(1,43 ; 1,93)$ & $1,77(1,48 ; 2,12)$ & $1,86(1,62 ; 2,14)$ & $1,99(1,68 ; 2,37)$ \\
\hline $55-59$ & $1,75(1,50 ; 2,03)$ & $1,84(1,53 ; 2,21)$ & $1,88(1,63 ; 2,16)$ & $2,04(1,71 ; 2,43)$ \\
\hline $60-64$ & $1,91(1,64 ; 2,23)$ & $1,87(1,55 ; 2,26)$ & $1,96(1,69 ; 2,27)$ & $1,89(1,57 ; 2,26)$ \\
\hline $65-69$ & $2,19(1,87 ; 2,57)$ & $2,32(1,90 ; 2,83)$ & $2,10(1,80 ; 2,44)$ & $2,21(1,83 ; 2,69)$ \\
\hline$\geq 70$ & $2,19(1,88 ; 2,54)$ & $2,16(1,78 ; 2,60)$ & $2,43(2,10 ; 2,80)$ & $2,24(1,87 ; 2,69)$ \\
\hline \multicolumn{5}{|l|}{ Escolaridade } \\
\hline Nenhuma & 1,00 & 1,00 & 1,00 & 1,00 \\
\hline Ensino fundamental incompleto & $0,66(0,63 ; 0,73)$ & $0,68(0,76 ; 0,90)$ & $0,60(0,33 ; 0,40)$ & $0,61(0,65 ; 0,77)$ \\
\hline Ensino fundamental completo & $0,64(0,59 ; 0,71)$ & $0,88(0,79 ; 0,98)$ & $0,55(0.50 ; 0,60)$ & $0,69(0,63 ; 0,77)$ \\
\hline Ensino médio & $0,47(0,42 ; 0,52)$ & $0,67(0,59 ; 0,76)$ & $0,36(0,34 ; 0,40)$ & $0,47(0,42 ; 0,53)$ \\
\hline Ensino superior & $0,39(0,32 ; 0,48)$ & $0,57(0,46 ; 0,70)$ & $0,30(0,25 ; 0,36)$ & $0,38(0,31 ; 0,47)$ \\
\hline \multicolumn{5}{|l|}{ Região do hospital } \\
\hline Sudeste & 1 & 1 & 1 & 1 \\
\hline Centro-0este & $1,21(1,02 ; 1,43)$ & $1,37(1,10 ; 1,71)$ & $1,32(1,13 ; 1,55)$ & $1,15(0,93 ; 1,42)$ \\
\hline Nordeste & $1,70(1,60 ; 1,81)$ & $1,64(1,52 ; 1,77)$ & $1,49(1,41 ; 1,58)$ & $1,26(1,17 ; 1,35)$ \\
\hline Norte & $1,84(1,69 ; 2,00)$ & $3,41(3,01 ; 3,86)$ & $1,38(1,27 ; 1,50)$ & $2,55(2,26 ; 2,89)$ \\
\hline Sul & $1,26(1,07 ; 1,26)$ & $1,42(1,25 ; 1,60)$ & $1,55(1,44 ; 1,67)$ & $1,93(1,72 ; 2,16)$ \\
\hline \multicolumn{5}{|l|}{ Unidade da Federação (UF) de nascimento } \\
\hline Igual à UF do tratamento & 1,00 & 1,00 & 1,00 & 1,00 \\
\hline Difere da UF do tratamento & $0,99(0,93 ; 1,05)$ & $0,93(0,86 ; 0,99)$ & $0,86(0,85 ; 0,95)$ & $0,90(0,83 ; 0,95)$ \\
\hline \multicolumn{5}{|l|}{ Tipo histológico } \\
\hline Epidermoides & 1,00 & 1,00 & 1,00 & 1,00 \\
\hline Adenocarcinomas & $0,62(0,57 ; 0,67)$ & $0,67(0,61 ; 0,74)$ & $0,46(0,43 ; 0,50)$ & $0,48(0,44 ; 0,52)$ \\
\hline Outros & $0,72(0,64 ; 0,82)$ & $0,74(0,63 ; 0,86)$ & $0,96(0,86 ; 1,08)$ & $0,97(0,84 ; 1,13)$ \\
\hline \multicolumn{5}{|l|}{ Ano do diagnóstico } \\
\hline $2000-2005$ & 1,00 & 1,00 & 1,00 & 1,00 \\
\hline 2006-2012 & $0,99(0,94 ; 1,04)$ & $0,99(0,92 ; 1,05)$ & $1,22(1,17 ; 1,29)$ & $1,23(1,16 ; 1,31)$ \\
\hline \multicolumn{5}{|l|}{ Raça/cor da pele } \\
\hline Branca & 1,00 & 1,00 & 1,00 & 1,00 \\
\hline Amarela & $1,05(0,81 ; 1,37)$ & $0,77(0,57 ; 1,03)$ & $1,00(0,78 ; 1,28)$ & $0,94(0,78 ; 1,28)$ \\
\hline Indígena & $1,49(0,85 ; 2,62)$ & $1,66(0,72 ; 3,81)$ & $2,08(1,24 ; 3,52)$ & $2,38(1,06 ; 5,33)$ \\
\hline Parda & $1,24(1,17 ; 1,31)$ & $0,91(0,84 ; 0,98)$ & $1,13(1,07 ; 1,19)$ & $0,99(0,92 ; 1,07)$ \\
\hline Preta & $1,00(0,89 ; 1,12)$ & $0,90(0,79 ; 1,03)$ & $1,19(1,07 ; 1,32)$ & $1,16(1,02 ; 1,31)$ \\
\hline \multicolumn{5}{|l|}{ Estado conjugal } \\
\hline Casada & 1,00 & 1,00 & 1,00 & 1,00 \\
\hline Separada & $0,92(0.82 ; 1,03)$ & $1,05(0,92 ; 1,20)$ & $1,16(1.04 ; 1,29)$ & $1,29(1,13 ; 1,46)$ \\
\hline Solteira & $0.98(0.92 ; 1,04)$ & $1,06(0,99 ; 1,14)$ & $1,28(1.20 ; 1,35)$ & $1,46(1,36 ; 1,57)$ \\
\hline Viúva & $1,36(1.26 ; 1,47)$ & $1,11(1,01 ; 1,23)$ & $1,66(1.57 ; 1,79)$ & $1,34(1,22 ; 1,48)$ \\
\hline
\end{tabular}

a) OR:odds ratio: razão de chances, calculada tendo a categoria 'doença inicial' como referência.

b) $\mathrm{IC}_{95 \%}$ : : 
estágio avançado da doença. Não foi possível identificar mudanças de tendência que pudessem suscitar hipóteses sobre efeitos da Portaria $\mathrm{n}^{0}$ 2.439/2005 do Ministério da Saúde.

As chances de desenvolver doença localizada e avançada no diagnóstico, cresceram paralelamente à idade, com tendência uniforme entre faixas etárias. Todavia há controvérsia na literatura com relação ao efeito da idade sobre estadiamento do câncer cervical. Evidências sugerem que uma menor aderência ao rastreamento, ${ }^{17}$ diferenças na história natural da doença (como um período pré-invasivo possivelmente mais curto) e alterações devidas à senescência do sistema imunológico podem estar relacionadas ao risco de diagnóstico em estágio avançado, entre a população idosa. ${ }^{18}$

0 estadiamento do câncer cervical na população estudada apresentou associação independente com a escolaridade, mesmo após o ajuste por outros fatores que poderiam atuar como confundidores. Estudo de coorte retrospectiva realizado na China, ${ }^{18}$ incluindo 68 mulheres, propõe modelo que incorpora fatores socioeconômicos, incluindo escolaridade, e características genéticas (tipagem do antígeno leucocitário humano) como fatores de risco para o desenvolvimento do câncer cervical. Estudo transversal com dados dos registros hospitalares de câncer no Brasil, relativo ao período de 2000 a 2009, analisou 37.638 casos de câncer cervical e concluiu que o diagnóstico do câncer cervical no país é feito tardiamente, em uma parcela expressiva das mulheres, além de relatar associação entre baixo nível educacional e estadiamento avançado. ${ }^{19}$

A relação entre baixa escolaridade e barreiras de acesso ao rastreamento do câncer cervical já foi apontada anteriormente. Inquérito incluindo informações de 1.214 mulheres em Belo Horizonte, $\mathrm{MG},{ }^{20}$ realizado no ano de 2008, observou que a baixa escolaridade, entre outros fatores, esteve associada a não aderência ao teste de rastreamento do câncer de colo uterino. $\mathrm{Ou}$ seja, mulheres com menor nível educacional estariam expostas a maior risco de ter diagnóstico tardio, em consonância com os resultados encontrados.

Análise da mortalidade por câncer cervical utilizando dados do Sistema de Informações sobre Mortalidade no Brasil, ${ }^{21}$ de 1980 a 2010, mostra tendência de aumento da mortalidade para câncer cervical em municípios fora das capitais das regiões Norte e Nordeste, ao contrário do encontrado para as demais regiões do país. Os resultados desse trabalho revelam, igualmente, aumento da mortalidade por câncer de mama nas mesmas regiões, contrariando a tendência de redução da mortalidade observada para as regiões Sul e Sudeste no mesmo período. Estes achados estão de acordo com o observado no presente estudo, exceto pelo fato de as mulheres tratadas na região Sul apresentarem chances maiores de diagnóstico em estágio avançado, na comparação com as mulheres da região Sudeste. Essa diferença pode estar relacionada à busca de atendimento por pessoas oriundas de regiões com maiores dificuldades de acesso aos serviços de saúde. ${ }^{22}$

Mulheres com diagnóstico histopatológico de adenocarcinoma apresentaram menores chances de serem diagnosticadas nos estágios mais avançados da doença, frente àquelas com tumores escamosos. Este achado contraria noção corrente na literatura, segundo a qual os adenocarcinomas, por se originarem mais frequentemente no canal endocervical, seriam diagnosticados mais tardiamente do que os tumores escamosos. ${ }^{23}$

Os resultados do presente estudo foram consistentes com aqueles de trabalho realizado no Reino Unido, ${ }^{24}$ em que foram incluídos 382 casos de câncer cervical com diagnóstico entre 1985 e 1996, e que apontou para tendência de estadiamento inicial para portadoras de adenocarcinomas. Esse tipo histológico também esteve associado a menor risco de apresentação em estágio avançado, segundo um trabalho com base em dados secundários sobre 51.842 mulheres no Brasil, de 2000 a $2009 .{ }^{25}$ Isso poderia explicar, ainda que parcialmente, o fato de se encontrar, em geral, menor benefício com a aplicação do rastreamento para adenocarcinomas. ${ }^{26}$

Mulheres negras e indígenas, assim como solteiras, separadas e viúvas, apresentaram maiores chances de desenvolver tanto doença localizada como avançada no diagnóstico, quando comparadas a mulheres brancas e casadas, mesmo após ajuste para o efeito da idade. Tal fato, em concordância com as evidências encontradas na literatura, indica a importância das barreiras sociais e do suporte familiar para uma adequada atenção à saúde da mulher. ${ }^{27-29}$ Há que se considerar, entretanto, a hipótese de confundimento residual no que se refere ao efeito da idade sobre as chances de diagnóstico avançado: mulheres viúvas tendem a ser mais idosas que as casadas e, portanto, é possível que parte do efeito encontrado seja devido à idade mais avançada.

As associações entre as caraterísticas analisadas e as categorias de desfecho mantiveram a mesma direção. 
Não obstante, há diferenças de magnitude dignas de nota. 0 efeito da região de tratamento sobre as chances de desenvolver doença localizada foi maior do que para doença avançada, entre mulheres tratadas em todas as regiões analisadas. Uma hipótese explicativa dessa associação seria a aplicação do rastreamento, levando a uma maior probabilidade de diagnóstico nas categorias inicial e localizada. ${ }^{30}$

Como pontos fortes do presente estudo, destaca-se o grande número de casos incluídos com representação de todos os estados do país. Cabe também destacar a metodologia utilizada: o modelo de regressão multinomial permitiu agrupar o estadiamento em categorias mais uniformes, agregando casos com prognóstico semelhante e facilitando a interpretação dos resultados. Estes fatores, em conjunto com a seleção rigorosa dos casos que garantiu a exclusão de tumores não invasivos, contribuíram para maior precisão dos resultados.

São resultados que apontam para uma associação entre baixo nível socioeconômico e estadiamento avançado de câncer cervical, principalmente entre mulheres negras, indígenas e de baixa escolaridade, no

\section{Referências}

1. Ferlay J, Soerjomataram I, Dikshit R, Eser S, Mathers C, Rebelo M, et al. Cancer incidence and mortality worldwide: Sources, methods and major patterns in GLOBOCAN 2012. Int J Cancer. 2015 Mar;136(5):E359-86.

2. Ministério da Saúde (BR). Instituto Nacional de Câncer José Alencar Gomes da Silva. Estimativa 2016: incidência de câncer no Brasil [Internet]. 2016 [citado 2015 nov 13]. Disponível em: http://www. inca.gov.br/estimativa/2016/.

3. International Agency for Research on Cancer. IARC handbooks of cancer prevention. Lyon: IARC Press; 2005. vol 10 .

4. Nascimento MI, Azevedo e Silva G. Effect of waiting time for radiotherapy on five-year overall survival in women with cervical cancer, 1995-2010. Cad Saúde Pública. 2015 Nov;31(11):2437-48.

5. Murillo R, Almonte M, Pereira A, Ferrer E, Gamboa OA, Jerónimo J, et al. Cervical cancer screening programs in Latin America and the Caribbean. Vaccine. 2008 Aug;26(Suppl 11):L37-48.

6. Brasil. Ministério da Saúde. Portaria GM nº 2.439, de 08 de dezembro de 2005. Institui a Política Nacional
Brasil. Análise específica da cadeia causal que norteia essas relações, assim como a identificação de possíveis confundidores não identificados nesta análise, seriam de grande valia na orientação de políticas públicas de saúde, necessárias e urgentes, capazes de reduzir essas desigualdades.

\section{Agradecimentos}

À Dra. Marise Rebelo, pela ajuda na obtenção e análise dos dados. Ao Dr. Gélcio Quintela Mendes e ao Dr. José Bines, pela orientação durante o processo de produção. À Dra. Andrea Negret, pela ajuda especializada nas traduções do idioma espanhol para o português.

\section{Contribuição dos autores}

Renna Junior NL e Azevedo e Silva G contribuíram na concepção do modelo teórico, coleta, preparo, análise e interpretação do banco de dados, redação e revisão crítica do manuscrito, aprovaram sua versão final e são responsáveis por todos os aspectos do trabalho, incluindo a garantia de sua precisão e integridade.

de Atenção Oncológica: promoção, prevenção, diagnóstico, tratamento, reabilitação e cuidados paliativos, a ser implantada em todas as unidades federadas, respeitadas as competências das três esferas de gestão. Diário Oficial da República Federativa do Brasil, Brasília (DF), 2005 dez 9; Seção 1:80.

7. Medeiros GC, Bergmann A, Aguiar SS, Thuler LCS. Análise dos determinantes que influenciam o tempo para o início do tratamento de mulheres com câncer de mama no Brasil. Cad Saúde Pública. 2015 jun;31(6):1269-82.

8. Thuler LCS, Bergmann A, Casado L. Perfil das pacientes com câncer do colo do útero no Brasil, 2000-2009: estudo de base secundária. Rev Bras Cancerol. 2012 jul-set;58(3):351-7.

9. Ministério da Saúde (BR). Instituto Nacional de Câncer. Registros hospitalares de câncer: planejamento e gestão [Internet]. 2 ed. Rio de Janeiro: Instituto Nacional de Câncer, 2010. 536 p. Disponível em: http://www1.inca.gov.br/inca/ Arquivos/INCA2009015_livro_registros_MIOLO.pdf.

10. Pecorelli S. Revised FIGO staging for carcinoma of the vulva, cervix, and endometrium. Int J Gynaecol Obstet. 2009 May;105(2):103-4. 
11. Kim HJ, Fay MP, Feuer EJ, Midthune DN. Permutation tests for joinpoint regression with applications to cancer rates. Stat Med. 2000 Feb;19(3):335-51.

12. Maldonado G, Greenland S. Simulation study of confounder-selection strategies. Am J Epidemiol. 1993 Dec;138(11):923-36.

13. Leifeld P. Conversion of statistical model output in R to LATEX and HTML Tables. J Stat Softw. 2013 Nov;55(8):1-24.

14. Ministério da Saúde (BR). Instituto Nacional de Câncer José Alencar Gomes da Silva. Informação dos registros hospitalares de câncer como estratégia de transformação: perfil do Instituto Nacional de Câncer José Alencar Gomes da Silva em 25 anos [Internet]. Rio de Janeiro: Instituto Nacional de Câncer José Alencar Gomes da Silva; 2012 [citado 2015 maio 3] 100 p. Disponível em: http://bvsms. saude.gov.br/bvs/publicacoes/inca/Informacao_dos_ registros_hospitalares.pdf

15. Brasil. Presidência da República. Casa Civil. Subchefia para Assuntos Jurídicos. Lei $\mathrm{n}^{0} 12.732$, de 22 de novembro de 2012. Dispõe sobre o primeiro tratamento de paciente com neoplasia maligna comprovada e estabelece prazo para seu início. Diário Oficial da República Federativa de Brasilia (DF), 2012 nov 23; Seção 1;1.

16. Vargas-Hernández VM, Vargas-Aguilar VM, TovarRodríguez JM. Primary cervical cancer screening. Fetección primaria del cancer cervicouterino. Cir Cir (English Edition). 2015 Sep;83(5):448-53.

17. Sawaya GF, Sung HY, Kearney KA, Miller M, Kinney W, Hiatt RA, et al. Advancing age and cervical cancer screening and prognosis. J Am Geriatr Soc. 2001 Nov;49(11):1499-504.

18. Hu B, Tao N, Zeng F, Zhao M, Qiu L, Chen W, et al. A risk evaluation model of cervical cancer based on etiology and human leukocyte antigen allele susceptibility. Int J Infect Dis. 2014 Nov;28:8-12.

19. Thuler LCS, Aguiar SS, Bergmann A. Determinantes do diagnóstico em estadio avançado do câncer do colo do útero no Brasil. Rev Bras Ginecol Obstet. 2014 Jun;36(6):237-43.

20. Lage AC, Pessoa MC, Meléndez JGV. Fatores associados à não realização do Teste de Papanicolaou em Belo Horizonte, Minas Gerais, 2008. REME Rev Min Enferm. 2013 jul-set;17(3):565-70.

21. Girianelli VR, Gamarra CJ, Azevedo e Silva G. Disparities in cervical and breast câncer mortality in Brazil. Rev Saúde Pública. 2014 Jun;48(3):459-67.
22. Gonzaga CMR, Freitas-Junior R, Barbaresco AA, Martins E, Bernardes BT, Resende PM. Cervical câncer mortality trends in Brazil: 1980-2009. Cad Saúde Pública. 2013 Mar;29(3):599-608.

23. Noh JM, Park W, Kim YS, Kim JY, Kim HJ, Kim J, et al. Comparison of clinical outcomes of adenocarcinoma and adenosquamous carcinoma in uterine cervical cancer patients receiving surgical resection followed by radiotherapy: A multicenter retrospective study (KROG 13-10). Gynecol Oncol. 2014 Mar;132(3):618-23.

24. Herbert A, Singh N, Smith JA. Adenocarcinoma of the uterine cervix compared with squamous cell carcinoma: a 12-year study in Southampton and South-west Hampshire. Cytopathology. 2001 Feb;12(1):26-36.

25. Nogueira-Rodrigues A, Ferreira CG, Bergmann A, de Aguiar SS, Thuler LCS. Comparison of adenocarcinoma (ACA) and squamous cell carcinoma (SCC) of the uterine cervix in a sub-optimally screened cohort: a population-based epidemiologic study of 51,842 women in Brazil. Gynecol Oncol. 2014 Nov;135(2):292-6.

26. Zappa M, Visioli CB, Ciatto S, Iossa A, Paci E, Sasieni P. Lower protection of cytological screening for adenocarcinomas and shorter protection for younger women: the results of a case-control study in Florence. Br J Cancer. 2004 May;90(9):1784-6.

27. Bairros FS, Meneghel SN, Dias-da-Costa JS, Bassani DG, Menezes AMB, Gigante DP, et al. Racial inequalities in access to women's health care in southern Brazil. Cad Saúde Pública. 2011 Dec;27(12):2364-72.

28. Ferrante JM, Gonzalez EC, Roetzheim RG, Pal N, Woodard L. Clinical and demographic predictors of late-stage cervical cancer. Arch Fam Med. 2000 May;9(5):439-45.

29. Schinkel JK, Zahm SH, Jatoi I, McGlynn KA, Gallagher C, Schairer C, et al. Racial/ethnic differences in breast cancer survival by inflammatory status and hormonal receptor status: an analysis of the surveillance, epidemiology, and end results data. Cancer Causes Control. 2014 Aug;25(8):959-68.

30. Sankaranarayanan R, Budukh AM, Rajkumar R. Effective screening programmes for cervical cancer in low- and middle-income developing countries. Bull World Health Organ. 2001 Jan;79(10):954-62. 


\section{Abstract}

Objective: To analyze the time trend and analyze the determinants of stage at diagnosis of cervical cancer in Brazil. Methods: Time trend analysis and cross-sectional study using data from hospital-based cancer registries (2000-2012); multinomial and joinpoint regression statistical models were used. Results: 65.843 cases were analyzed; the median interval between diagnosis and treatment was 59 days; the percentage of advanced staging increased, annual percent change 1.10\% (95\% CI 0.80;1.50); women with higher education (compared to unlettered) had less odds of late stage diagnosis $(O R=0.38 ; 95 \%$ CI 0.31;0.47); among indigenous $(O R=2.38 ; 95 \%$ CI 1.06;5.33) and black women $(O R=1.16$; 95\%CI 1.02;1.31), compared to white, and in the North region (OR $=2.55 ; 95 \%$ CI 2.26;2.89), compared to the Southeast, the odds was higher; other factors positively related to advanced stage were 'age', 'bistology', and marital status'. Conclusion Inequities affect the odds of late stage diagnosis of cervical cancer.

Keywords: Uterine Cervical Neoplasms; Women's Health; Hospital Records; Health Services Accessibility; Medical Oncology.

\section{Resumen}

Objetivo: estudiarlatendenciatemporaly determinantes asociados al diagnóstico en estado avanzado de cáncer cervical en Brasil. Métodos: análisis de tendencias de tiempoy estudio transversal utilizando datos de los registros bospitalarios de cáncer (2000-2012); se utilizaron modelos de regresión multinomialy joinpoint. Resultados: 65.843 casos fueron analizados; la mediana del intervalo entre diagnóstico y tratamiento fue 59 días; el porcentaje de casos con estado avanzado aumentó, variación porcentual anual $1,10\left({ }_{95 \%}\right.$ 0,8;1,50); mujeres con educación superior (en comparación con no letradas) tenían menos probabilidad de diagnóstico tardio $\left(O R=0,38 ; I_{95 \%} 0,31 ; 0,47\right)$; indígenas $\left(O R=2,38 ; I C_{95 \%} 1,06 ; 5,33\right)$ y negras $\left(O R=1,16 ; I C_{95 \%}\right.$ 1,02;1,31), en comparación con blancas, y mujeres tratadas en la región Norte $\left(O R=2,55 ; I C_{95 \%}, 2,26 ; 2,89\right)$, comparadas con Sudeste, presentaron mayor probabilidad; otros factores relacionados con estadio avanzado fueron 'edad', 'histología' y 'estado civil'. Conclusión: las desigualdades afectan el diagnóstico tardio de cáncer cervical.

Palabras-clave: Neoplasias del Cuello Uterino; Salud de la Mujer; Registros de Hospitales; Accesibilidad a los Servicios de Salud; Oncología Médica.

Recebido em 28/08/2017

Aprovado em 30/11/2017 